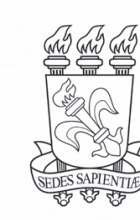

\title{
Artigos
}

\section{Impacto antrópico na dinâmica de uma floresta nebular do planalto catarinense}

\author{
Anthropic impact on the dynamics of a cloud forest in the planalto \\ catarinense region
}

\section{Giovana Reali Stuani ${ }^{\oplus}{ }^{\circ}$ Ana Carolina da Silva' ${ }^{\oplus}$, Pedro Higuchi ${ }^{\oplus}$, Janaina Gabriela Larsen ${ }^{\oplus}$, Felipe Domingos Machado ${ }^{\oplus}$, Guilherme Neto dos Santos ${ }^{\circledR}$}

'Universidade do Estado de Santa Catarina, Lages, SC, Brasil

\section{RESUMO}

O objetivo do presente estudo foi investigar a dinâmica da comunidade arbórea adulta e regenerante em um fragmento de Floresta Nebular em Urubici, Santa Catarina, sujeito ao impacto de gado. Os inventários dos indivíduos arbóreos adultos (diâmetro na altura do peito - DAP $\geq 5 \mathrm{~cm}$ ) foram realizados em 2011, 2015 e 2019, e dos regenerantes arbóreos (DAP < 5 cm), em 2013, 2015, 2017 e 2019. Para o componente adulto, a amostragem foi realizada em 25 parcelas de $20 \times 20 \mathrm{~m}$ alocadas de forma sistemática no fragmento. Em cada uma dessas parcelas também foram coletadas variáveis edáficas e topográficas. O componente regenerativo arbóreo foi avaliado em subparcelas conforme classe de tamanho das plantas (classe 1: plantas com altura de $0,15 \mathrm{~cm}$ até $1 \mathrm{~m}$, em $5 \mathrm{~m}^{2}$; classe 2: plantas com altura acima de $1 \mathrm{~m}$ e até $3 \mathrm{~m}$, em $10 \mathrm{~m}^{2}$, e classe 3: plantas com altura maior que $3 \mathrm{~m}$ e DAP $<5 \mathrm{~cm}$, em $20 \mathrm{~m}^{2}$ ). Foram calculadas as taxas demográficas para a comunidade e as populações de indivíduos adultos e regenerantes. Utilizaram-se Árvores de Regressão (AR), para testar a influência das variáveis ambientais sobre as taxas de dinâmica dos regenerantes, e Análises dos Componentes Principais (PCA), para sintetizar os gradientes representados pelas taxas de dinâmica entre populações. No último período, 2015-2019, a floresta apresentou perda em indivíduos e em área basal, o que sugere um processo de desestruturação. Para os indivíduos regenerantes, as variáveis mais influentes na dinâmica estiveram relacionadas com a fertilidade do solo, os teores de $\mathrm{H}+\mathrm{Al}$, a cota altitudinal e a compactação dos solos. Para a conservação da área, medidas, como o cercamento contra entrada de gado, são essenciais para a manutenção da floresta ao longo do tempo.

Palavras-chave: Dinâmica florestal; Floresta Ombrófila Mista; Impacto do gado; Análise multivariada 


\section{ABSTRACT}

The objective of this research was to investigate the dynamics of the adult and regenerating tree community in a Cloud Forest fragment in Urubici, Santa Catarina, subject to livestock impact. Inventories of adult tree individuals were carried out in 2011, 2015, and 2019, and of regenerative trees, in $2013,2015,2017$, and 2019. For the adult component, sampling was performed within $2520 \times 20$ $\mathrm{m}$ systematically allocated plots. Within each plot, soil and topographic variables were also collected. The regenerative component was evaluated in subplots according to plant size classes (class 1: plants with a height from $0.15 \mathrm{~cm}$ to $1 \mathrm{~m}$ in $5 \mathrm{~m}^{2}$; class 2: plants with height above $1 \mathrm{~m}$ and up to $3 \mathrm{~m}$ in 10 $\mathrm{m}^{2}$; and class 3: plants with height above $3 \mathrm{~m}$ and DAP $<5 \mathrm{~cm}$ in $20 \mathrm{~m}^{2}$ ). The demographic rates for the community of adult and regenerating individuals were determined. Regression Trees (RT) were used to test the influence of environmental variables on the dynamics rates of the regenerative component, and Principal Component Analysis (PCA) was performed to synthesize the population dynamics rates. In the last period, 2015-2019, the forest showed a loss of individuals and basal area, which suggests a de-structuring process. For regenerating individuals, the most influential variables in the dynamics were soil fertility, $\mathrm{H}+\mathrm{Al}$ levels, altitudinal quota, and soil compaction. For the conservation of the area, measures such as fencing to prevent cattle from entering are essential for the maintenance of the forest over time.

Keywords: Forest dynamics; Subtropical mix forest; Cattle impact; Multivariate analysis

\section{INTRODUÇÃO}

As florestas nebulares ocorrem em elevadas altitudes, em que a nebulosidade é frequente. Estas são encontradas na região do Planalto Sul Catarinense, na borda da Serra Geral, em locais cuja altitude ultrapassa, frequentemente, os 1.400 m, podendo, assim, ser classificadas, segundo o IBGE (2012), como Floresta Ombrófila Mista (FOM) Alto-Montana. Esta fitofisionomia se caracteriza por possuir neblina durante a maior parte do tempo, formando um microclima peculiar, com elevada umidade relativa do ar e baixa radiação solar global. Devido às suas condições climáticas específicas, florestas nebulares apresentam um alto grau de endemismo (PONCE-REYES et al., 2012) e são de suma importância para a manutenção da qualidade e quantidade de água, em decorrência da presença de nascentes de rios (GOLDSMITH; MATZKE; DAWSON, 2013).

Destaca-se que, especialmente no século XX, assim como toda Floresta Ombrófila Mista, essa formação vegetacional passou por um histórico processo de degradação, relacionado ao corte seletivo de espécies de interesse madeireiro, tais 
Stuani, G. R.; Silva, A. C.; Higuchi, P.; Larsen, J. G.; Machado, F. D.; Santos, G. N. | 1716

como a Araucaria angustifolia (Bertol.) Kuntze e a imbuia (Ocotea porosa (Nees) L. Barr.), e à expansão de áreas agropecuárias (SEVEGNANI et al., 2019). Atualmente, a paisagem natural encontra-se fragmentada, com remanescentes apresentando diferentes níveis de degradação (SEVEGNANI; VIBRANS; GASPER, 2013). Além disso, a proximidade entre essas áreas florestais remanescentes e campos utilizados para a produção de gado de corte facilita a entrada de animais no interior dos remanescentes florestais, causando impactos, como o pisoteio, a compactação do solo e a herbivoria de plântulas (ETCHEBARNE; BRAZEIRO, 2016).

Até recentemente, poucos estudos sobre a dinâmica demográfica de comunidades de espécies arbóreas em parcelas permanentes foram realizados em áreas altomontanas, no domínio da Floresta Atlântica (DALLABRIDA et al., 2017; FERREIRA et al., 2017; TERRA et al., 2018). De forma geral, os resultados encontrados nesses trabalhos reforçam a ideia de que, em escala local, a complexa interação entre fatores edáficos e o regime de distúrbio são determinantes nos padrões de dinâmica, com o favorecimento de espécies pioneiras em locais com maior fertilidade do solo e sujeitos a um histórico mais intenso de perturbações. Esse é um aspecto crítico do ponto de vista de conservação de remanescentes florestais, tendo em vista que a continuidade de impactos antrópicos ao longo do tempo tem o potencial de efeitos negativos sobre a biodiversidade, como, por exemplo, aqueles relacionados com a homogeneização biológica (LÔBO et al., 2011).

Considerando a elevada importância dos remanescentes de florestas nebulares, assim como os impactos negativos aos quais estão susceptíveis, estudos são necessários para a detecção, qualificação e quantificação desses distúrbios, a fim de gerar resultados que podem servir como base para a definição de estratégias de conservação. Dentro desse contexto, objetivou-se investigar a dinâmica da comunidade arbórea adulta e regenerante em um fragmento de Floresta Nebular em Urubici, no Planalto Catarinense, sujeito à presença de gado. 


\section{MATERIAL E MÉTODOS}

\subsection{Caracterização da área de estudo}

O estudo foi realizado em um fragmento de Floresta Nebular, de 346 ha, localizado no município de Urubici, no Planalto Serrano Catarinense, na latitude $28^{\circ} 04^{\prime} \mathrm{S}$ e longitude $49^{\circ} 37^{\prime} \mathrm{O}$, com altitude aproximada de $1.600 \mathrm{~m}$. A região possui clima Cfb, de acordo com a classificação de Köppen, com temperatura média anual de $15^{\circ} \mathrm{C}$ a $16^{\circ} \mathrm{C}$, com ocorrência de geada e neve no inverno. A precipitação média anual está entre 1.200 e 1.900 mm, com chuvas bem distribuídas durante o ano (INSTITUTO NACIONAL DE METEOROLOGIA, 2019).

A fitofisionomia do fragmento pode ser classificada, segundo o IBGE (2012), como Floresta Ombrófila Mista Alto-Montana. A presença de nuvens constantes também o caracteriza como uma área de floresta nebular. Os solos da região são pouco profundos, classificados, principalmente, como Neossolos Litólicos e Cambissolos (MARCON et al., 2014). O fragmento florestal estudado encontra-se bem estruturado e pode ser classificado como de estágio sucessional avançado. A área pertence a uma propriedade particular, na qual existe influência de gado tanto na borda como no interior da floresta, resultando em impactos causados pelos bovinos, como a presença de fezes, pisoteio e herbivoria.

\subsection{Coleta de dados}

\subsubsection{Componente adulto}

Para o estudo das espécies arbóreas deste fragmento florestal, foram alocadas parcelas permanentes que permitem o monitoramento ao longo do tempo. A composição e a estrutura do componente arbóreo adulto foram inicialmente caracterizadas por Marcon et al. (2014), que, em 2011, alocaram 25 parcelas

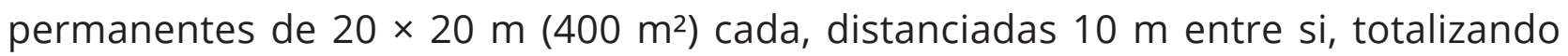


1 ha de área amostrada. Em cada parcela, os autores mensuraram, identificaram e marcaram com plaquetas os indivíduos arbóreos que apresentaram circunferência na altura do peito (CAP), medido a 1,30 m do solo, igual ou superior a 15,7 cm. Aqueles que possuíam troncos múltiplos foram medidos quando a raiz da soma dos quadrados dos CAPs foi maior do que $15,7 \mathrm{~cm}$.

Em 2015 (SANTOS et al., 2021) e em 2019 (presente estudo), foram realizados estudos de dinâmica nas mesmas parcelas, utilizando-se a mesma metodologia. Para isso, dentro das parcelas, os indivíduos sobreviventes foram remedidos, os indivíduos que atingiram valores de inclusão de 15,7 cm de CAP foram considerados recrutas e identificados, mensurados e plaqueteados, e os indivíduos mortos foram contabilizados.

\subsubsection{Componente regenerante}

O componente regenerante foi avaliado, pela primeira vez, por Dalla Rosa et al. (2016), em 2013. Os autores alocaram, no interior de cada uma das 25 parcelas do componente adulto, subparcelas de $20 \mathrm{~m}^{2}(20 \times 1 \mathrm{~m})$, subdivididas de acordo com o tamanho dos regenerantes (ver VEFAGO et al., 2019). Todos os indivíduos regenerantes arbóreos foram identificados, mensurados e plaqueteados, conforme os seguintes critérios: classe 1, com plantas com altura entre $15 \mathrm{~cm}$ e $1 \mathrm{~m}$, foi avaliada em $5 \mathrm{~m}^{2}$ (5 × $1 \mathrm{~m}$ ); classe 2, com plantas com altura maior que $1 \mathrm{~m}$ e até $3 \mathrm{~m}$, foi avaliada em $10 \mathrm{~m}^{2}$ $(10 \times 1 \mathrm{~m})$, e classe 3 , com plantas com altura maior que $3 \mathrm{~m}$ e DAP menor que $5 \mathrm{~cm}$, foi avaliada em $20 \mathrm{~m}^{2}$ (20 × 1 m). Em 2015 (SANTOS et al., 2021) e nos anos 2017 e 2019 (ambos os anos referentes ao presente estudo), repetiu-se o inventário conforme a metodologia anterior, quando foram recrutados os indivíduos arbóreos regenerantes que atingiram os valores de inclusão, sendo identificados, inseridos em classes de altura e plaqueteados. Os indivíduos sobreviventes foram mensurados novamente e os indivíduos mortos foram contabilizados. 


\subsubsection{Caracterização ambiental}

Para a caracterização ambiental da área de estudo, foram utilizados dados edáficos e topográficos coletados por Marcon et al. (2014). Os autores caracterizaram as propriedades físico-químicas do solo de cada parcela, quantificando $\mathrm{pH}$, fósforo $(\mathrm{P})$, potássio $(\mathrm{K})$, cálcio $(\mathrm{Ca})$, magnésio $(\mathrm{Mg})$, alumínio $(\mathrm{Al})$, teor de matéria orgânica $(\mathrm{MO})$, teores de hidrogênio e alumínio $(\mathrm{H}+\mathrm{Al})$, capacidade de troca catiônica efetiva (CTC), saturação por base na CTC efetiva (V) e os teores de areia, silte e argila. Também determinaram o grau de compactação do solo nas parcelas, na profundidade de 0 a $20 \mathrm{~cm}$, obtendo-se o valor médio de compactação ao longo do perfil avaliado, compactação máxima e compactação a $20 \mathrm{~cm}$. Ainda, realizaram o levantamento topográfico, obtendo-se a cota altimétrica, o desnível máximo e a declividade média.

\subsubsection{Análise de dados}

Para ambos os componentes, foram obtidas as taxas de recrutamento (1) e mortalidade (2), rotatividade (3) e mudança líquida (4), em número de indivíduos. Para a regeneração natural, ainda foram obtidas as taxas de mudança para classes superiores (5) e inferiores de altura (6); e, para o componente arbóreo adulto, as taxas de perda em área basal (7) e ganho (8), assim como a mudança líquida (9) e a rotatividade (10) em área basal (ver GROSS et al., 2018; VEFAGO et al., 2019).

$$
\begin{aligned}
& M=\left\{1-\left[\frac{\left(n_{0}-m\right)}{n_{0}}\right]^{\frac{1}{t}}\right\} \times 100 \\
& R=\left[1-\left(1-\frac{r}{n_{t}}\right)^{\frac{1}{t}}\right] \times 100 \\
& \text { Rot ind }=\frac{M+R}{2} \\
& \text { ML ind }=\left[\left(\frac{n_{t}}{n_{0}}\right)^{\frac{1}{t}}-1\right] \times 100
\end{aligned}
$$


$M S=\left\{1-\left[\frac{\left(n_{0}-m s\right)}{n_{0}}\right]^{\frac{1}{t}}\right\} \times 100$

$M I=\left\{1-\left[\frac{\left(n_{0}-m i\right)}{n_{0}}\right]^{\frac{1}{t}}\right\} \times 100$

$P A B=\left\{1-\left[\frac{\left(A B_{0}-\left(A B_{m}+A B_{d e c}\right)\right)}{A B_{0}}\right]^{\frac{1}{t}}\right\} \times 100$

$G A B=\left[1-\left(1-\frac{\left(A B_{r}+A B_{\text {cresc }}\right)}{A B_{t}}\right)^{\frac{1}{t}}\right] \times 100$

$\operatorname{Rot} A B=\frac{P A B+G A B}{2}$

$M L A B=\left[\left(\frac{A B_{t}}{A B_{0}}\right)^{\frac{1}{t}}-1\right] \times 100$

Em que: $\mathrm{M}=$ Taxa de mortalidade $\left(\% \cdot \mathrm{ano}^{-1}\right) ; \mathrm{N}_{\mathrm{o}}=$ número de indivíduos no primeiro inventário; $\mathrm{m}=$ número de indivíduos mortos entre inventários; $\mathrm{t}=$ tempo entre inventários (anos); $\mathrm{R}=$ Taxa de recrutamento $\left(\% \cdot \mathrm{ano}^{-1}\right) ; r=$ número de indivíduos recrutados entre inventários; $n_{\mathrm{t}}=$ número de indivíduos no último inventário; Rot ind = Taxa de rotatividade de número de indivíduos (\%.ano-1); $\mathrm{ML}$ ind = Taxa de mudança líquida em número de indivíduos $\left(\% \cdot a n 0^{-1}\right) ; \mathrm{MS}=$ Taxa de mudança para classes de tamanho superiores (\%.ano-1 $)$; ms = número de indivíduos que passaram para classes de tamanho superiores; $\mathrm{MI}=$ Taxa de mudança para classes de tamanho inferiores (\%.ano1); $\mathrm{mi}=$ número de indivíduos que passaram para classes de tamanho inferiores; $\mathrm{PAB}$ = Taxa de perda em área basal (\%.ano-1); $\mathrm{AB}_{0}=$ Área basal no primeiro inventário $\left(\mathrm{m}^{2}\right)$; $A B_{m}=$ Área basal de árvores mortas $\left(m^{2}\right) ; A B_{\text {dec }}=$ decremento em área basal das árvores sobreviventes $\left(\mathrm{m}^{2}\right) ; \mathrm{GAB}=$ Taxa de ganho em área basal $\left(\% . \mathrm{ano}^{-1}\right) ; \mathrm{AB}_{\mathrm{r}}=$ Área basal das árvores recrutadas $\left(m^{2}\right) ; A B_{\text {cresc }}=$ crescimento em área basal das árvores sobreviventes $\left(\mathrm{m}^{2}\right)$; Rot $\mathrm{AB}=$ Taxa de rotatividade em área basal $\left(\% \cdot a \mathrm{no}^{-1}\right)$ e $\mathrm{ML} \mathrm{AB}=$ Taxa de mudança líquida em área basal (\%.ano-1).

Utilizaram-se Árvores de Regressão (AR) para testar a influência das variáveis ambientais sobre as taxas de dinâmica do componente regenerante, tendo, como variáveis independentes, as variáveis ambientais, e como variáveis dependentes, as taxas de dinâmica. Como critério de poda das Árvores de Regressão, foi utilizada a redução do parâmetro de complexidade (CP). A PCA (Análise dos Componentes Principais) foi utilizada para sintetizar os gradientes representados pelas taxas de dinâmica dos componentes arbóreo regenerante e adultos. Para isso, os dados foram escalados para terem a média centralizada em zero e variância unitária. 
Todas as análises foram realizadas por meio da linguagem de programação estatística R (R CORE TEAM, 2018).

\section{RESULTADOS E DISCUSSÃO}

A riqueza de espécies observada ao longo dos anos, tanto para o componente adulto (34, 35 e 31) quanto para o regenerante (29, 33, 32 e 31), caracterizou-se por uma relativa estabilidade, sugerindo que, em um curto período de tempo (<10 anos) e na ausência acentuada de distúrbios de larga escala, essa propriedade da vegetação não apresente grandes modificações em formações florestais tardias. Ainda, os valores encontrados são semelhantes aos observados em outros fragmentos florestais altomontanos da região (DUARTE et al., 2018; SÜHS et al., 2019). Porém, quando comparados aos valores referentes às áreas situadas em menores pisos altitudinais, a riqueza pode ser considerada baixa (SANTOS et al., 2018). Isso reforça a ideia de elevada seletividade ambiental das florestas de altitude, em função, predominantemente, do clima, caracterizado por baixa temperatura e alta nebulosidade (LONG et al., 2014). Como destacado por Oliveira-Filho et al. (2015), a ocorrência de geadas, evento frequente na região de estudo, é um forte fator limitante ao estabelecimento de espécies arbóreas, principalmente aquelas de caráter mais tropical, o que explica a baixa riqueza relativa observada no presente estudo.

Quanto à abundância de indivíduos arbóreos adultos, observou-se uma relativa estabilidade no primeiro período (2011 = $1.580 \pm 15,51$ ind.; $2015=1.601 \pm 15,51$ ind.), seguida por uma expressiva redução no último ano (2019 = $1.442 \pm 16,01$ ind.). Para a área basal, após um incremento de $42,0\left( \pm 0,31 \mathrm{~m}^{2}\right)$ para $44,21 \mathrm{~m}^{2}\left( \pm 0,33 \mathrm{~m}^{2}\right)$, no primeiro período, a floresta apresentou uma redução para $42,07 \mathrm{~m}^{2}\left( \pm 0,39 \mathrm{~m}^{2}\right)$, em 2019. Esses resultados refletem os padrões demográficos do componente arbóreo, em que, para o período de 2011-2015, as taxas de recrutamento (0,9\%.ano $\left.{ }^{-1}\right)$ e de ganho em área basal $(2,03 \%$.ano-1) foram superiores às taxas de mortalidade $(0,57 \%$.ano-1 $)$ e perda em área basal (0,77 \%.ano-1 ). No período seguinte (2015-2019), ocorreu o inverso, com maiores valores de mortalidade (3,23 \%.ano-1) e perda em área basal (3,57 \%.ano $\left.{ }^{-1}\right)$ do que de recrutamento $\left(0,67 \%\right.$.ano $\left.{ }^{-1}\right)$ e ganho em área basal $(2,37 \%$.ano-1) (Figura $1 \mathrm{~A})$. 
Figura 1 - Taxas demográficas (mortalidade, recrutamento, ganho e perda em área basal - $A B)$, em \%.ano-1, para indivíduos arbóreos adultos $(A)$ e da regeneração natural (B) de um fragmento de floresta nebular em Urubici-SC, sob impacto de gado

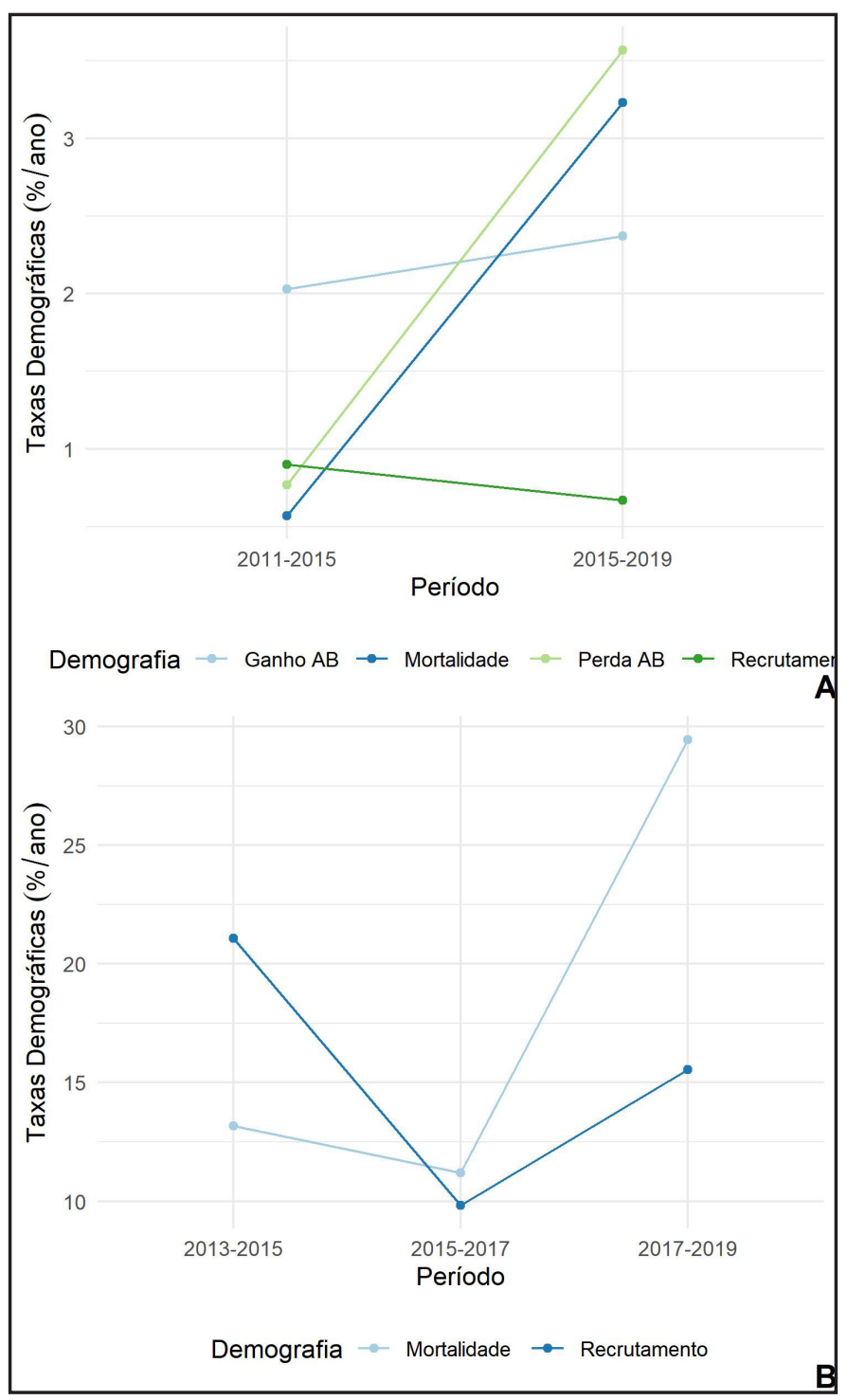

Fonte: Autores (2020)

Para os indivíduos regenerantes, que representam parte do estoque das árvores futuras da floresta, após o incremento em abundância no primeiro intervalo (2013 = $524 \pm 12,18$ ind.; $2015=634 \pm 13,08$ ind.), os anos subsequentes foram caracterizados por acentuada redução de indivíduos, principalmente em 2019 (2017 = $615 \pm$ 11,94 ind.; $2019=429 \pm 10,28$ ind.). Essa variação temporal expressa as taxas demográficas dos regenerantes, em que, com exceção do primeiro intervalo (2013-2015), observase a mortalidade excedendo o recrutamento de regenerantes (Figura 1B). Nesse 
sentido, destaca-se o período de 2017-2019, quando a mortalidade (29,50 \%.ano-1) foi expressivamente superior ao recrutamento (15,50 \%.ano-1). De fato, nesse ano, foram observados, dentro da floresta, indícios de maior presença de gado, com trilhas e áreas pisoteadas, assim como muitas árvores caídas, possivelmente por causa de eventos climáticos mais extremos, como tempestades acompanhadas por ventos.

Assim, as taxas demográficas evidenciam uma mudança no padrão silvigenético ao longo do tempo (MACHADO; OLIVEIRA-FILHO, 2010), de forma que uma fase de estruturação, com incremento em abundância e área basal, foi sucedida por uma fase de desestruturação, com redução em abundância e em área basal. Dessa forma, em contraposição à relativa estabilidade da riqueza florística, destaca-se a instabilidade estrutural da floresta avaliada. Flutuações na dinâmica ao longo do tempo são comuns, no entanto, não se pode descartar a possibilidade de efeitos negativos de impactos antropogênicos crônicos, como aqueles relacionados à presença de gado, cujos principais impactos estão associados à compactação do solo e herbivoria (ETCHEBARNE; BRAZEIRO, 2016). Nas áreas de floresta onde há maior pastoreio, pode ter ocorrido impacto sobre as populações mais sensíveis a distúrbios desta natureza (ETCHEBARNE; BRAZEIRO, 2016), influenciando, assim, nas taxas de mortalidade e de recrutamento dos regenerantes arbóreos.

Apesar dos resultados gerais observados para a comunidade ao longo do tempo, destaca-se que as diferentes populações apresentaram padrões distintos (Figuras 2 e 3). Na PCA para o componente adulto, o Eixo 1, que explicou 57,4\% da inércia total (Figura 2A), sintetizou um gradiente relacionado a rotatividade e perda em área basal (Figura 2B). Enquanto espécies, como Myrceugenia glaucescens (Cambess.) D.Legrand \& Kausel, Myrceugenia miersiana (Gardner) D. Legrand \& Kausel e Drimys angustifolia Miers, apresentaram elevada rotatividade, mortalidade e perda em área basal, Ilex microdonta Reissek, Ocotea pulchella (Nees) Mez e Ilex paraguariensis A. St. -Hil. tenderam a apresentar padrões opostos, com baixa rotatividade e reduzidas taxas de mortalidade e perda em área basal. O Eixo 2 da mesma ordenação, que explicou 28,7\% da inércia total, sintetizou um gradiente de mudança líquida e de recrutamento, com Myrrhinium atropurpureum Schott e Drimys angustifolia se destacando com maiores incrementos em número de indivíduos e área basal, e Myrceugenia myrcioides (Cambess.) O. Berg e Dicksonia sellowiana Hook. apresentando maior redução em abundância e área basal. 
Figura 2 - Ordenação das espécies na Análise de Componentes Principais (A) e contribuição das variáveis para os eixos 1 (B) e 2 (C), para os indivíduos adultos amostrados em um fragmento de floresta nebular em Urubici-SC, sob impacto de gado


Contribution of variables to Dim-2

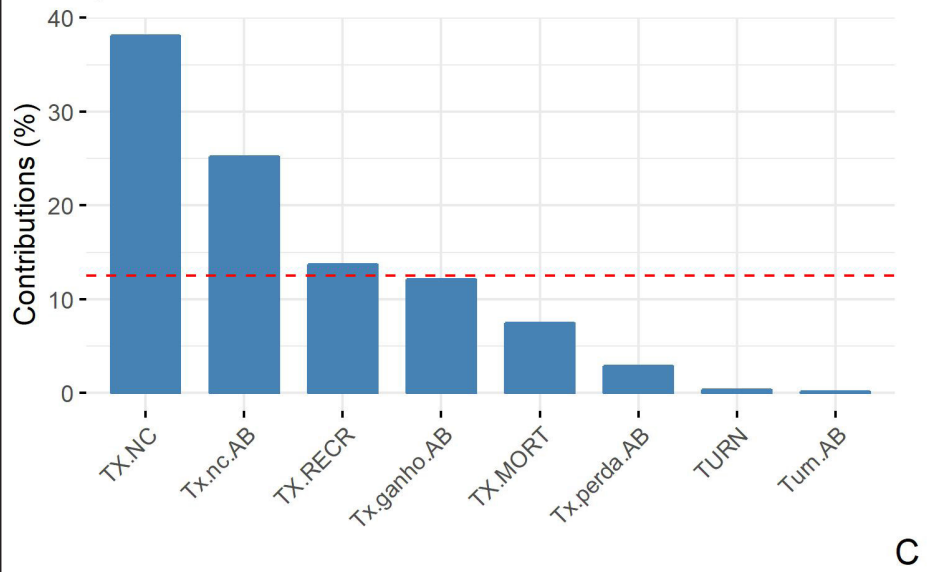

Fonte: Autores (2020)

Onde: Tx.nc.AB = taxa de mudança líquida em área basal; TX.NC = taxa de mudança líquida em número de indivíduos; TX.RECR = taxa de recrutamento; Tx.ganho. $A B=$ taxa em ganho da área basal; TURN = rotatividade em número de indivíduos; Turn. $A B=$ taxa de rotatividade em área basal; Tx.perda.AB = taxa de perda de área basal; TX.MORT = taxa de mortalidade. 
Para o componente da regeneração natural (Figura 3A), o Eixo 1 explicou 49,6 \% dos dados, enquanto o Eixo 2 explicou cerca de 28,3 \%. As taxas demográficas mais correlacionadas com o Eixo 1 foram a mortalidade, a rotatividade em número de indivíduos e o recrutamento (Figura 3B). Para o Eixo 2, as taxas mais importantes foram a mudança líquida em número de indivíduos, o recrutamento, a mudança para classe superior de altura e a mudança para classe inferior de altura (Figura 3C). À direita da ordenação, estão as espécies com maiores taxas de mortalidade, rotatividade e recrutamento, como Symphyopappus itatiayensis (Hieron.) R.M. King \& H. Rob., Prunus myrtifolia (L.) Urb. e Ilex paraguariensis St. Hill. À esquerda do gráfico, estão as espécies com características antagônicas, como Myrceugenia glauscecens (Cambess.) D. Legrand \& Kausel. Acima do gráfico, estão espécies com elevada mudança líquida, mas também com elevadas taxas de mudanças para classes superiores e inferiores de altura, como Myrceugenia miersiania (Gardner) D. Legrand \& Kausel e Myrceugenia euosma (O.Berg) D. Legrand; e abaixo do gráfico, as espécies com características contrárias, como Ocotea pulchella (Nees) Mez., Myrceugenia regnelliana (O.Berg) D. Legrand \& Kausel, Citronella paniculata (Mart.) Howard e Symplocos uniflora (Pohl) Benth.

Os diferentes padrões demográficos observados entre as populações refletem as diferentes estratégias ecológicas e as respostas das mesmas frente às condições locais (SILVA et al., 2017). Nesse sentido, observa-se que enquanto algumas espécies tenderam a aumentar a representatividade na comunidade (e.g., Myrrhinium atropurpureum e Drimys angustifolia, nos adultos, e Myrceugenia miersiania e Myrceugenia euosma, na regeneração), evidenciando estarem bem adaptadas às condições locais, outras, ao contrário, tenderam a reduzir a presença na comunidade (e.g., Ocotea pulchella, Myrceugenia regnelliana, Citronella paniculata e Symplocos uniflora). 
Figura 3 - Ordenação das espécies na Análise de Componentes Principais (A) e contribuição das variáveis para os Eixos 1 (B) e 2 (C), para os indivíduos regenerantes amostrados em um fragmento de floresta nebular em Urubici-SC, sob impacto de gado
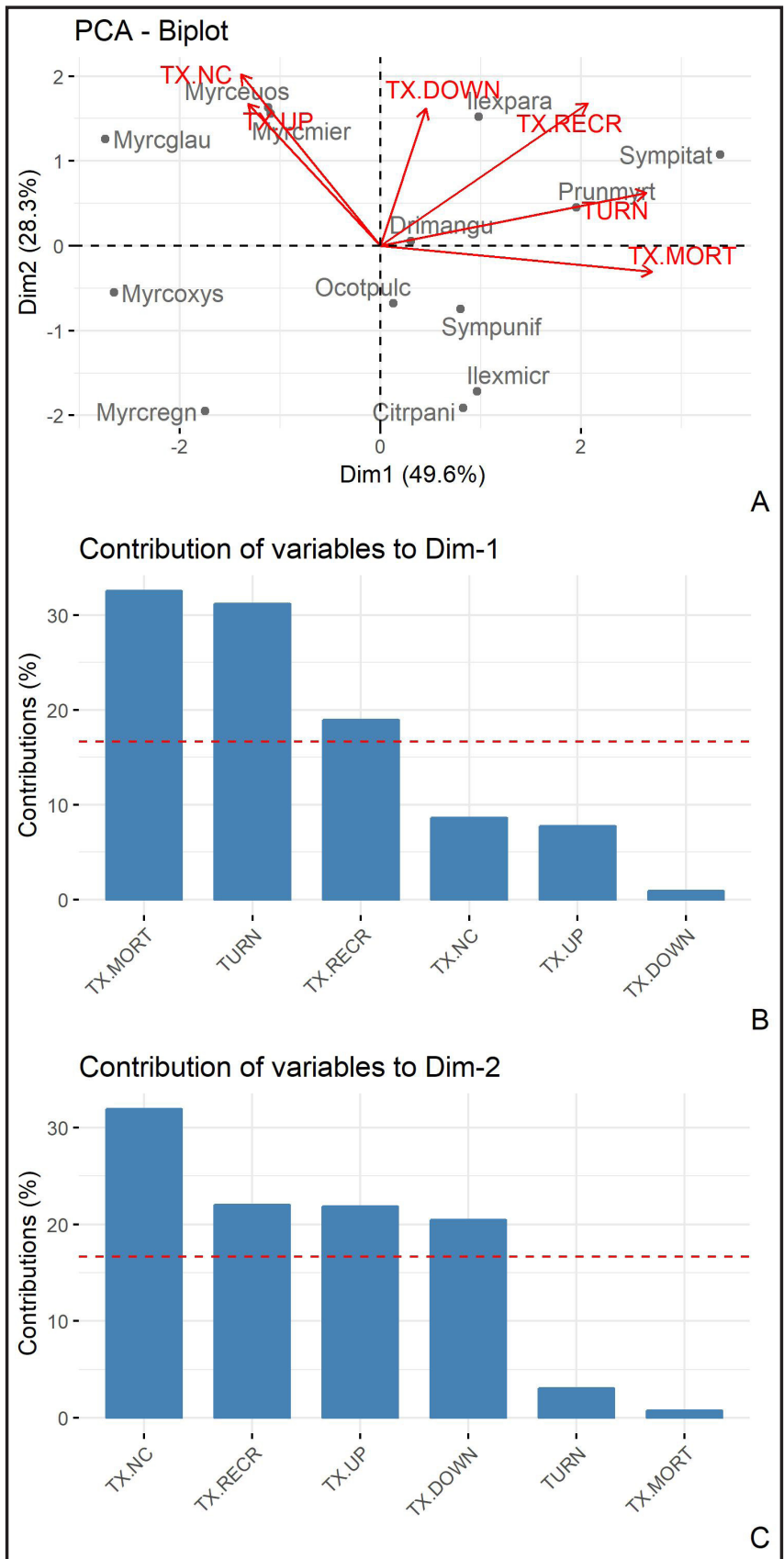

Fonte: Autores (2020)

Onde: TX. NC = taxa de mudança líquida em número de indivíduos; TX.UP = taxa de mudança para classe superior de altura; TX.DOWN = taxa de mudança para classe inferior de altura; TX.RECR = taxa de recrutamento; TURN = taxa de rotatividade em número de indivíduos; TX.MORT = taxa de mortalidade. 
Destaca-se que, para a regeneração natural, componente cujas respostas a distúrbios são relativamente mais perceptíveis em uma escala temporal de curto prazo ( $<2$ anos), apenas as taxas de mortalidade, recrutamento, mudanças para classes superiores e mudança líquida foram influenciadas de forma significativa pelas variáveis ambientais (Figura 4). A mortalidade foi maior em locais com menores teores de Ca no solo (Figura 4A), o recrutamento foi maior em parcelas localizadas em menores altitudes (Figura 4B), a mudança para classes de tamanho superiores foi maior em solos com menores valores $\mathrm{H}+\mathrm{Al}$ (Figura 4C) e as mudanças líquidas positivas foram observadas em locais com solos menos compactados (Figura 4D).

Figura 4 - Árvore de Regressão para as taxas de mortalidade (A), recrutamento (B), mudança para classe superior de altura (C) e mudança líquida em número de indivíduos (D) da regeneração natural em função das variáveis ambientais

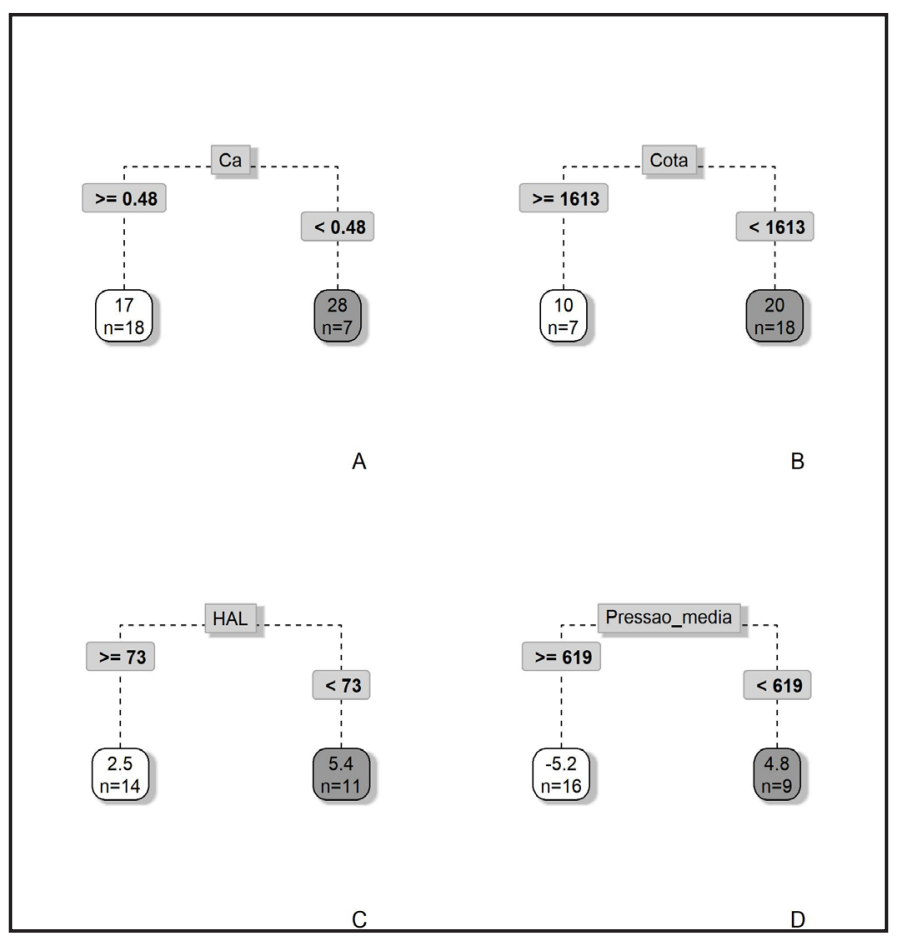

Fonte: Autores (2020)

Onde: $\mathrm{n}$ = quantidade de subparcelas que se enquadram em cada condição ambiental; $\mathrm{Ca}=$ teores de cálcio; Cota = valor de cota altimétrica; HAL = teores de alumínio e hidróxido no solo; pressão média = compactação do solo médio no perfil de até $20 \mathrm{~cm}$ de profundidade. 
Tomados em conjunto, esses resultados sugerem o caráter limitante de solos menos férteis (<Ca), ácidos (>H+Al) e compactados (> Pressão Média) para regeneração, que, nestas circunstâncias, tende a apresentar maior mortalidade, menor crescimento em altura e mudanças líquidas negativas. Esses resultados corroboram com outros já encontrados na literatura, evidenciando a importância de fatores edáficos sobre o padrão de regeneração natural de espécies arbóreas (TOLEDO et al., 2011; BENTOS; NASCIMENTO; WILLIAMSON, 2013). Em particular, destacam-se os efeitos negativos da compactação do solo, que representa um fator de degradação relacionado a atividades antropogênicas, como a exploração seletiva de madeira e a presença de gado (HATTORI et al., 2013; BORGES; CALONEGO; ROSOLEM, 2019).

\section{CONCLUSÕES}

Infere-se que os distúrbios pelos quais a floresta em avançado estágio sucessional passou nos últimos anos, tais como a presença de gado, se refletem em sua dinâmica estrutural, com risco de degradação da floresta, a qual apresentou, no último período, perda em indivíduos e em área basal. Para os indivíduos regenerantes, representantes de parte do estoque das árvores futuras, as variáveis ambientais mais influentes na dinâmica estiveram relacionadas com a fertilidade do solo, os teores de $\mathrm{H}+\mathrm{Al}$, a cota altitudinal e a compactação dos solos, sendo esta última um indicativo da presença do gado. Para a conservação da área, medidas, como o cercamento contra a entrada de gado, são essenciais para a manutenção da floresta ao longo do tempo. Essa recomendação pode ser estendida para áreas de APP (Área de Preservação Permanente) e Reserva Legal das florestas naturais na região. Ainda, por meio do presente trabalho, foi possível detectar as espécies que estão contribuindo mais para as taxas de dinâmica, tanto por meio de sua sensibilidade aos impactos (e.g. Prunus myrtifolia na regeneração, com elevada mortalidade), como as que apresentam provável resiliência a estes (e.g. Ilex paraguariensis na regeneração, com elevado crescimento). Destaca-se a necessidade de ampliação de estudo com esta natureza, considerando outras localidades e condições ambientais, de forma que possa extrair padrões mais conclusivos e gerais sobre os fenômenos observados localmente neste trabalho. 


\section{AGRADECIMENTOS}

À FAPESC pelo suporte financeiro por meio do PAP/UDESC. Ao CNPq pela concessão de bolsas aos três primeiros autores.

\section{REFERÊNCIAS}

BENTOS, T. V.; NASCIMENTO, H. E. M.; WILLIAMSON, G. B. Tree seedling recruitment in Amazon secondary forest: Importance of topography and gap micro-site conditions. Forest Ecology and Management, Amsterdam, v. 287, p. 140-146, 2013.

BORGES, W. L.; CALONEGO, J. C.; ROSOLEM, C. A. Impact of crop-livestock-forest integration on soil quality. Agroforestry Systems, New York, v. 92, p. 2111-2119, 2019.

DALLABRIDA,J.P. etal. Tree component demography in an Upper Montane Mixed Ombrophilous Forest under chronic anthropogenic disturbances. Revista Árvore, Viçosa, MG, v. 41, n. 3, 2017.

DALLA ROSA, A. et al. Regeneração natural de espécies arbóreas em uma floresta nebular em Santa Catarina. Revista Árvore, Viçosa, MG, v. 40, n. 6, p. 1073-1082, 2016.

DUARTE, E. et al. Sucessão em fragmentos florestais altomontanos no Sul do Brasil: uma abordagem florístico-estrutural e filogenética. Ciência Florestal, Santa Maria, v. 28, p. 898912, 2018.

ETCHEBARNE, V.; BRAZEIRO, A. Effects of livestock exclusion in forests of Uruguay: soil condition and tree regeneration. Forest Ecology and Management, Amsterdam, v. 362, p. 120-129, 2016.

FERREIRA, P. I. et al. Dinâmica de bracatingais inequiâneos sob diferentes condições ambientais no Planalto Sul Catarinense. Floresta, Curitiba, v. 47, n. 1, p. 43-54, 2017.

GOLDSMITH, G. R.; MATZKE, N. J.; DAWSON, T. R. The incidence and implications of clouds for cloud forest plant water relations. Ecology Letters, Hoboken, v. 16, n. 3, p. 307-314, 2013.

GROSS, A. et al. Fragmentation as a key driver of tree community dynamics in mixed subtropical evergreen forests in Southern Brazil. Forest Ecology and Management, Amsterdam, v. 411, p. 20-26, 2018.

HATTORI, D. et al. Effects of soil compaction on the growth and mortality of planted dipterocarp seedlings in a logged-over tropical rainforest in Sarawak, Malaysia. Forest Ecology and Managment, Amsterdam, v. 310, p. 770-776, 2013.

IBGE. Manual Técnico da Vegetação Brasileira. Rio de Janeiro, 2012. 
INSTITUTO NACIONAL DE METEOROLOGIA (Brasil). Gráficos climatológicos. Brasília, 2019. Disponível em: http://www.inmet.gov.br/portal/index.php?r=clima/graficosClimaticos. Acesso em: 07 nov. 2019.

LÔBO, D. et al. Forest fragmentation drives Atlantic forest of northeastern Brazil to biotic homogenization. Diversity and Distributions, Hoboken, v. 17, n. 2, p. 287-296, 2011.

LONG, W. et al. Community assembly in a tropical cloud forest related to specific leaf area and maximum species height. Journal of Vegetation Science, Hoboken, v. 26, n. 3, p. 513-523, 2014.

MACHADO, E. L. M.; OLIVEIRA-FILHO, A. T. Spatial patterns of tree community dynamics are detectable in a small (4 ha) and disturbed fragment of the Brazilian Atlantic forest. Acta Botanica Brasílica, Belo Horizonte, v. 24, p. 250-261, 2010.

MARCON, A. K. et al. Variação florístico-estrutural em resposta à heterogeneidade ambiental em uma Floresta Nebular em Urubici, Planalto Catarinense. Scientia Forestalis, Piracicaba, v. 42, n. 103, p. 439-450, 2014.

OLIVEIRA-FILHO, A. T. et al. Delving into the variations in tree species composition and richness across South American subtropical Atlantic and Pampean forests. Journal of plant ecology, Oxford, v. 8, n. 3, p. 242-260, 2015.

PONCE-REYES, R. et al. Vulnerability of cloud forest reserves in Mexico to climate change. Nature Climate Change, New York, v. 2, p. 448-452, 2012.

R CORE TEAM. R: a language and environment for statistical computing. [S. I.]: R Foundation for Statistical Computing, 2018. Disponível em: http://www.r-project.org. Acesso em: 30 jul. 2018.

SANTOS, G. N. et al. Dinâmica do componente arbóreo adulto e regenerante em uma floresta nebular no Planalto Sul Catarinense. Ciência Florestal, Santa Maria, v. 3, n.3, p. 1086-1104, 2021.

SANTOS, G. N. et al. Regeneração natural em uma floresta com araucária: inferências sobre o processo de construção da comunidade de espécies arbóreas. Ciência Florestal, Santa Maria, v. 28, n. 2, p. 483-494, 2018.

SEVEGNANI, L. et al. Structure and diversity of the Araucaria forest in southern Brazil: biotic homogenisation hinders the recognition of floristic assemblages related to altitude, Southern Forests: a Journal of Forest Science, Abingdon-on-Thames, v. 81, n. 4, p. 297-305, 2019.

SEVEGNANI, L.; VIBRANS, A. C.; GASPER, A. L. Considerações sobre a Floresta Ombrófila Mista em Santa Catarina. In: VIBRANS, A. C. et al (ed.). Inventário Florestal de Santa Catarina, Floresta Ombrófila Mista. Blumenau, Edifurb, 2013. v. 3.

SILVA, M. A. M. et al. Traits and functional strategies as predictors of demographic variations over a chronosequence. Brazilian Journal of Botany, New York, n. 40, p. 761-770, 2017. 
SÜHS, R. B. et al. Species diversity, community structure and ecological traits of trees in an upper montane forest, southern Brazil. Acta Botanica Brasilica, Belo Horizonte, v. 33, n. 1, p. 153-162, 2019.

TERRA, M. C. N. S. et al. Short-term changes in an ombrophilous atlantic forest. Nativa: Pesquisas Agrárias e Ambientais, Sinop, v. 6, n. 5, p. 543-550, 2018.

TOLEDO, J. J. et al. How much variation in tree mortality is predicted by soil and topography in Central Amazonia? Forest Ecology and Management, Amsterdan, v. 263, n. 3, p. 331-338, 2011.

VEFAGO, M. B. et al. What explains thevariation on the regenerative component dynamics of Araucaria Forests in southern Brazil. Scientia Agricola, Piracicaba, v. 76, p. 405-414, 2019.

\section{Contribuição de Autoria}

\section{1 - Giovana Reali Stuani}

Engenheira Florestal

https://orcid.org/0000-0003-3212-5484• giovanarealistuani@gmail.com

Contribuição: Investigação, Visualização, Escrita - primeira redação, Escrita - revisão e edição

\section{2 - Ana Carolina da Silva}

Engenheira Florestal, Dra., Professora

https://orcid.org/0000-0002-1285-640X·carol_sil4@yahoo.com.br

Contribuição: Conceituação, Curadoria de dados, Aquisição de financiamento, Metodologia, Administração de projetos, Supervisão, Visualização, Escrita - revisão e edição

\section{3 - Pedro Higuchi}

Engenheiro Florestal, Dr., Professor

https://orcid.org/0000-0002-3855-555X•higuchip@gmail.com

Contribuição: Análise formal, Investigação, Metodologia, Escrita - revisão e edição

\section{4 - Janaina Gabriela Larsen}

Engenheira Florestal

https://orcid.org/0000-0002-5603-5208•janainalarsen18@gmail.com

Contribuição: Investigação, Visualização, Escrita - revisão e edição 


\section{5 - Felipe Domingos Machado}

Engenheiro Florestal

https://orcid.org/0000-0002-1731-4730•felipe.d.machado@hotmail.com

Contribuição: Investigação, Visualização, Escrita - revisão e edição

\section{6 - Guilherme Neto dos Santos}

Engenheiro Florestal

https://orcid.org/0000-0003-0346-6941•guineto.florestal@gmail.com

Contribuição: Investigação, Visualização, Escrita - revisão e edição

\section{Como citar este artigo}

Stuani, G. R.; Silva, A. C.; Higuchi, P.; Larsen, J. G.; Machado, F. D.; Santos, G. N. Impacto antrópico na dinâmica de uma floresta nebular do planalto catarinense. Ciência Florestal, Santa Maria, v. 31, n. 4, p. 1714-1732, 2021. DOI 10.5902/1980509842667. Disponível em: https://doi. org/10.5902/1980509842667. 